\title{
DC Arc Fault Detection Methods in MEA Distribution Systems
}

\author{
Jeffy Thomas, Rory Telford, Puran Rakhra, Patrick Norman, and Graeme Burt
}

University of Strathclyde

\begin{abstract}
Direct current (DC) for primary power distribution is a promising solution that is being explored by aircraft system integrators for MEA applications to enable the paralleling of non-synchronized engine off-take generators, and to enable the reduction of energy conversion stages required to supply electronically actuated loads. However, a significant challenge in the use of DC systems is the reliable detection of arc faults. Arcing presents a significant fire risk to aircraft and their presence can result in critical system damage and potentially fatal conditions. Series arc faults in DC systems are particularly challenging to detect as the associated reduction in system current eliminates the use of conventional overcurrent and current differential methods for fault detection. This paper provides an overview of series arc faults in DC systems and presents both simulation and hardware results to illustrate key trends, characteristics and discriminating features. It also presents a comprehensive review of arc fault detection and diagnosis techniques that have been proposed for a wide range of aerospace and other applications. The paper concludes with a discussion on the unique challenges and opportunities for the application of both deterministic and probabilistic methods in MEA systems.
\end{abstract}

\section{Introduction}

The replacement of hydraulically and pneumatically powered nonpropulsive loads with electrical equivalents on future more-electric aircraft (MEA) will require a higher-capacity electrical power distribution systems (EPS), integrated with advanced power electronic conversion and protection technologies, arranged to form highlyresilient network architectures. Direct current (DC) for primary power distribution is a promising solution that is being explored by aircraft system integrators for MEA applications as it enables the paralleling of non-synchronized engine off-take generators and reduces the number of energy conversion stages required to supply electronically actuated loads [1]. Other benefits include higher reliability, voltage stability and improved power quality [2] in comparison to equivalent alternating current (AC) systems. However, a significant challenge present in the migration towards DC primary power systems is the development of highly reliable and discriminative series arc fault protection systems.

Given the extreme environmental conditions that aircraft electrical systems are subjected to, series arc faults may arise due to the vibration of loose terminal connections or as degraded wires contact metal structures, and so are typically intermittent in nature [3]. In an AC system, the arc is normally extinguished at the natural zero-crossing of the current profile whilst re-ignition is subject to the electrode gap length and ionisation conditions. However, DC series arc faults are particularly aggressive due to the lack of natural zero-crossings in the current profile [4], and so may remain exposed for prolonged periods of time if not rapidly detected and isolated. The heat generated during these events presents significant fire risk to exposed aircraft subsystems, impacting overall system safety and reliability. Furthermore, the reduction of fault current when series arcing occurs eliminates the use of conventional overcurrent and current differential methods for protection, and so alternative methods for detection and isolation are required.

This paper first discusses the formation of series arc faults and identifies the characteristics of their behaviour that make them particularly challenging to detect. Key frequency domain features extracted from simulated and experimental arc fault data that may be exploited for novel detection and discrimination methods are then presented, with an overview of both the hardware testbed and software models used to generate the data. A comprehensive literature review of arc fault detection methods is then undertaken to establish the stateof-the-art methods used across a wide range of power system applications, and considers the applicability of such methods for future MEA applications. The paper concludes with a discussion on the potential challenge of certifying non-deterministic arc fault detection methods for aircraft applications and discusses the merit and feasibility of achieving a purely deterministic arc fault detection system for future DC aircraft power systems.

\section{Arc Fault Formation}

Electrical arcs are formed when ionization occurs in air gaps between conductors [5] and, when voltage across the anode and cathode exceeds the dielectric breakdown voltage, arc current discharges across the air gap. Regular arcing may occur during mechanical switching events - these types of arcs are highly transient, and circuit breakers (CBs) and other mechanical switching devices are designed to withstand their formation. Conversely, arc current through ionized gas during fault events may be fully sustained, and the resultant heat generated during such events presents a significant risk of fire to surrounding insulation. Many conditions may cause an arc fault, including: deterioration of wiring and interconnections; loose terminal connections and wiring damaged during routine maintenance [3].

Arc faults are categorised as either parallel or series. Parallel faults occur between two phase conductors or a phase conductor and ground. These faults are parallel to the load and usually considered to be a form of low impedance short circuit. Series faults often begin with corrosion in pin-socket connections or loose connections in series with electrical loads. A particularly challenging characteristic of series arc fault behaviour that makes them difficult to detect is the effective reduction in load current as a result of the additional series impedance introduced to the circuit from the formation of the arc itself. Accordingly, current may actually fall below rated levels and well below relay trip curves, 
eliminating the use of conventional overcurrent and current differential methods for protection. Previous studies [6] have demonstrated that a single intermittent arc event may last for approximately $1.25 \mathrm{~ms}$, with a series of events extending to 30ms [6]. However, there are limited studies on arcing characteristics in DC systems, and the lack of a zero crossing coupled intermittency caused by in-flight vibration often adds complexity to detection.

The development of technology for detecting and isolating $\mathrm{AC}$ arc fault conditions is relatively mature in comparison to DC faults. For example, arc fault circuit interrupters (AFCI) are commercially deployed and are typically limited to AC distribution. However, future aircraft EPS are likely to utilise increased elements of DC distribution owing to the advantages offered at a systems level [2], [7]. The nature of AC supplies results in distinct features at zero-crossings during fault conditions, supporting fault discrimination [4]. Conversely, DC series arcing is likely to be more sustained as there is a lack of zero-crossings, making extracting discriminative features for detection purposes more challenging.

An increase in dynamic loads in future MEA EPS will further compound the protection challenge as detection systems will require the ability to accurately discriminate between true fault events and highly transient load changes. Moreover, detection may be further complicated by the effect that higher altitude conditions have on arc formation and characteristics. These factors, coupled with the migration towards DC systems in future aircraft power systems, are motivation for the continued research and development of highly reliable and discriminative series DC arc fault detection systems.

\section{Characteristic Features of Series DC Arc Faults}

Arc fault detection can be realised by identifying characteristic features extracted from current and voltage profiles as the arc forms. Figure 1 illustrates example profiles of a DC series arc, obtained from [8], as the fault develops into a fully open circuit condition. Figure 1 highlights a sustained arcing condition, where load current decreases and arc voltage increases non-linearly after the intitial drop during fault onset. This is a result of arc length increasing as the conductor separate.
However, such features of arc elongation may not be present, or at least as pronounced, during intermittent conditions.

Although these DC arc fault profiles exhibit seemingly unique characteristic features, such as a negative rate-of-change of current $(d i / d t)$ upon fault inception, the development of voltage across the arc, and the presence of high frequency noise, discriminating between fault conditions and nominal system events, such as load switching, is challenging. A distinguishing feature between series and parallel faults is the initial decrease in load current under series conditions. Given that load current decreases as the arc initiates, overcurrent thresholdbased statistical features in the time domain are not effective for their detection. Furthermore, measurement of voltage drop across sections of electrical networks is less common. Therefore, it is typical for detection systems to use features extracted from either the frequency or time-frequency [9] domain of current profiles.

The following case study identifies key frequency domain characteristics of series arcing through the analysis of synthesised arc fault data. Fault generation was conducted in both simulation and hardware environments, and the associated arc currents were transformed to the frequency domain using the Fast Fourier Transform (FFT).

\section{Simulation of Series DC Arcing}

A validated series arc fault model, originally developed by Uriarte et al. [10] for DC microgrid applications, was utilised for the purpose of simulating electromagnetic transient behaviour as an arc fault is generated. The model, built using the MATLAB/Simulink platform, exhibits the stochastic nature of the arc while accounting for intermittency, quenching attempts and energy consumption under loading parameters. The model characterises the arc voltage, $V_{\text {gap }}$, and current, $I_{g a p}$, to account for exponential resistance, $R_{g a p}$. Similar principles where used when designing the hardware set-up, a representative diagram is illustrated in Figure 2.
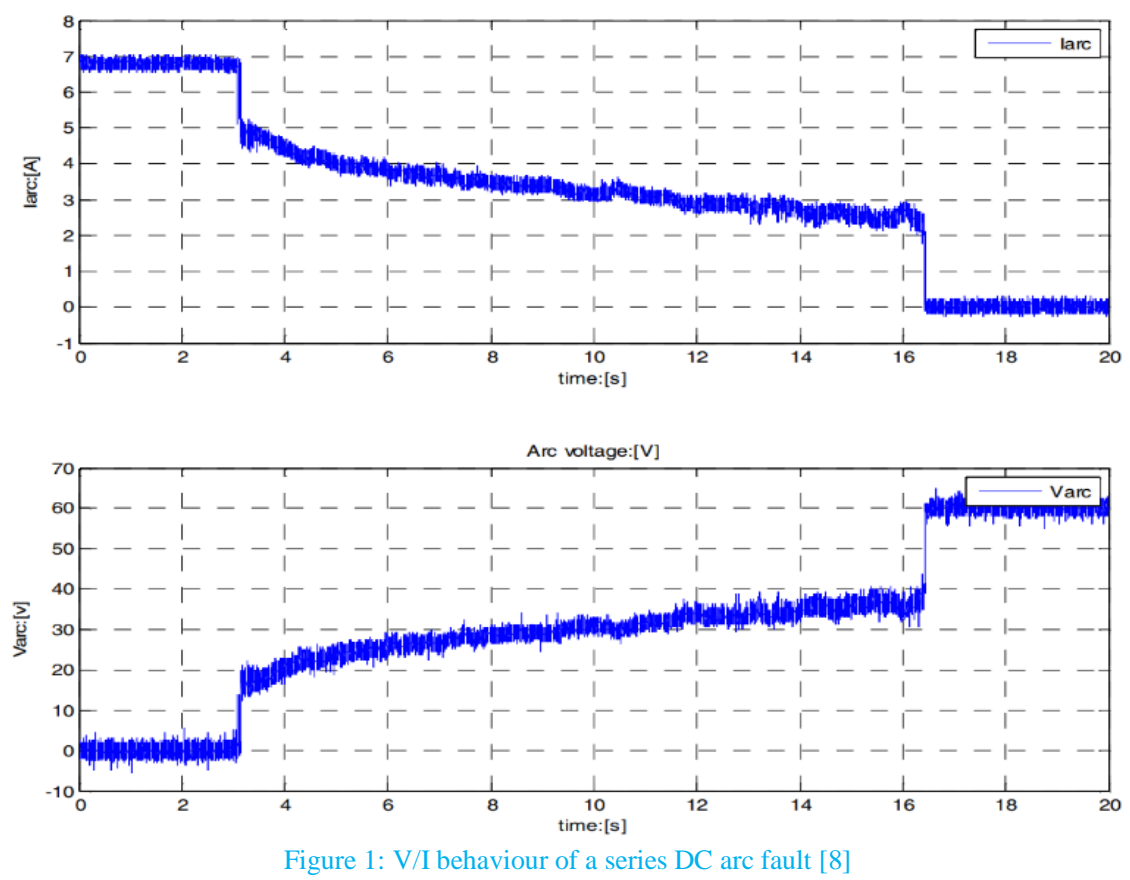

Page 2 of 11 
Load Current Measurement Bank

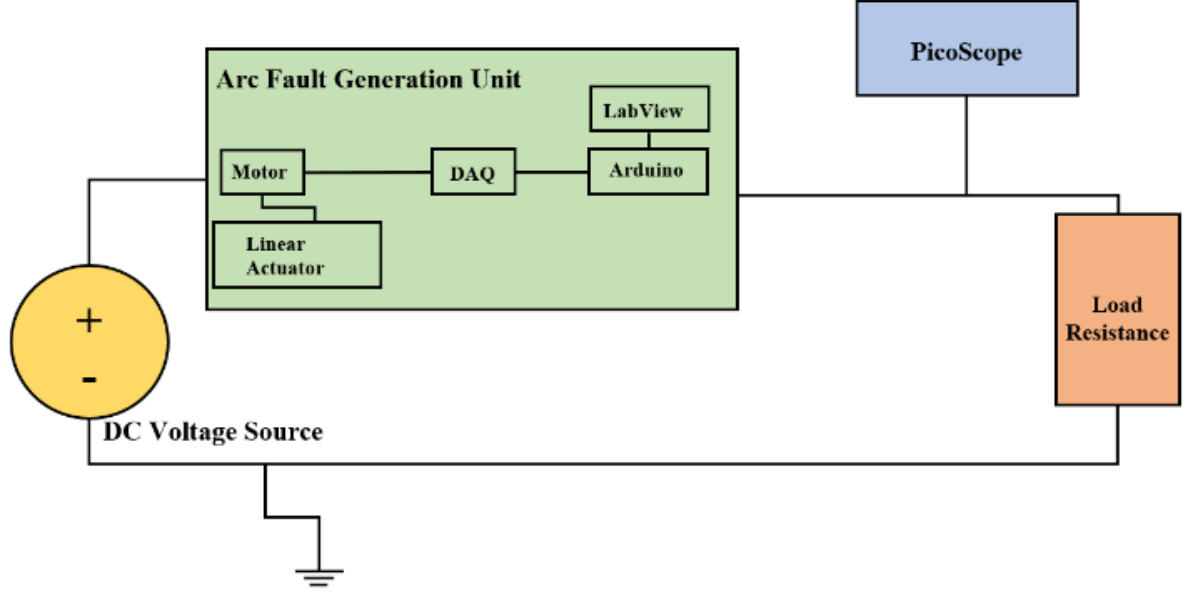

Figure 2: Design of the hardware set-up of arc current measurement

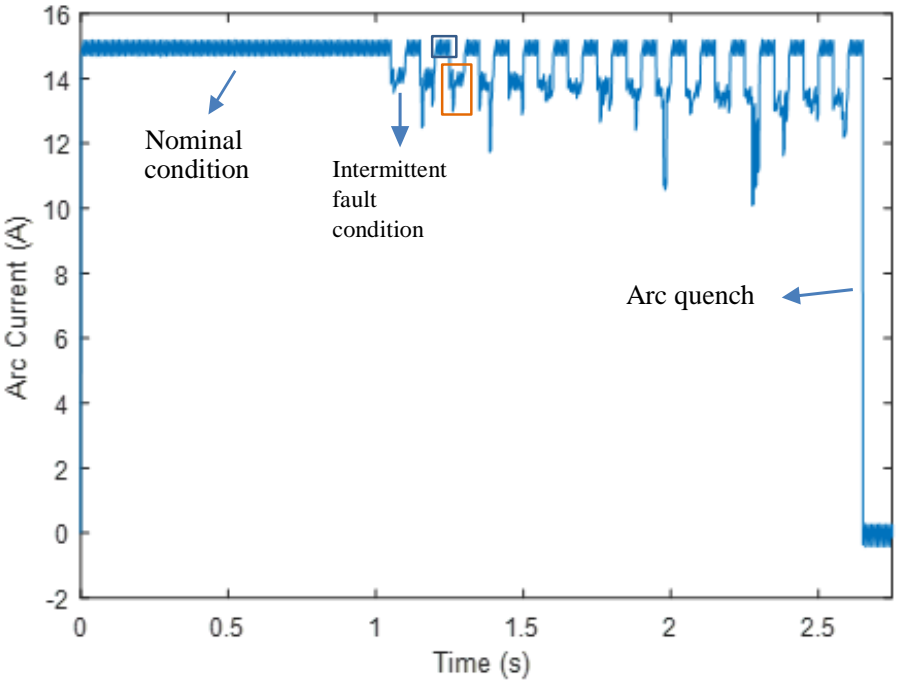

Figure 3: Simulated arc current waveform

The random characteristic of the arc is accounted for through a time variance in $R_{g a p}$. The model is valid for a voltage range of $280 V_{d c}$ to $800 V_{d c}$. The advantage of this particular model is that no prior knowledge of plasma, thermal or power system conditions is required to simulate the arc. The simulation model consists of a power supply block, series arc fault emulator, load current measurement and a load bank to emulate a power distribution system. The developed model enables preliminary analysis on the arc fault data to be conducted to observe trends and behaviours at different ranges of frequency bands. The extraction of these trends and behaviours may then be applied to a real testbed dataset for comparison.

Figure 3 shows the simulated load current behaviour under both nominal and fault conditions. The arc fault is introduced after 1 second of simulation time with intermittent behaviour observed between 1 and 2.6 seconds followed by the onset of an arc quench. The sustained intermittency of the waveform can be attributed to the conductors being in temporary discontinuous contact. After 2.6 seconds of

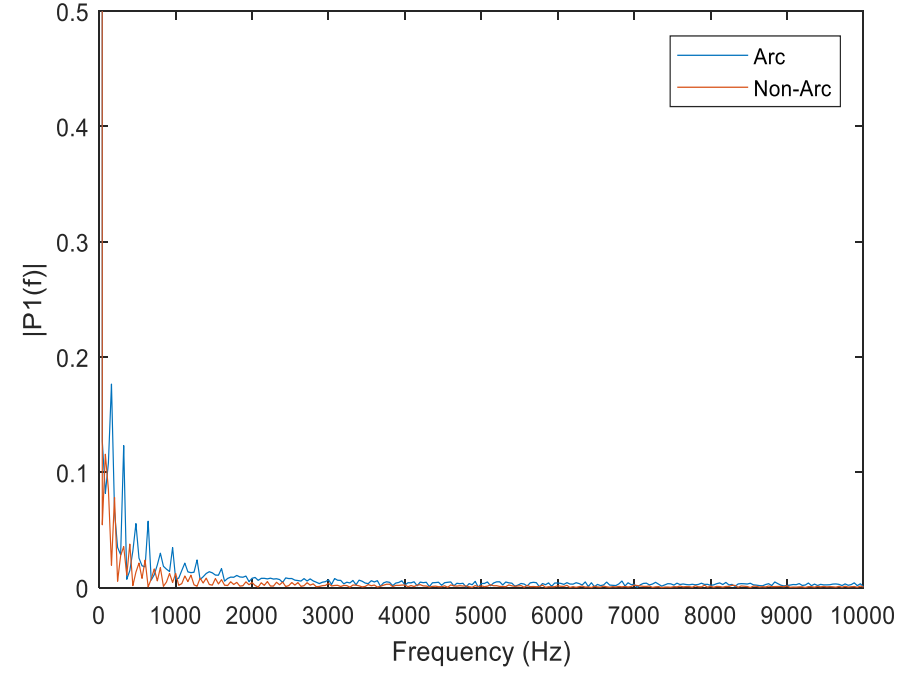

Figure 4: FFT amplitude spectrum of simulated arc current waveform

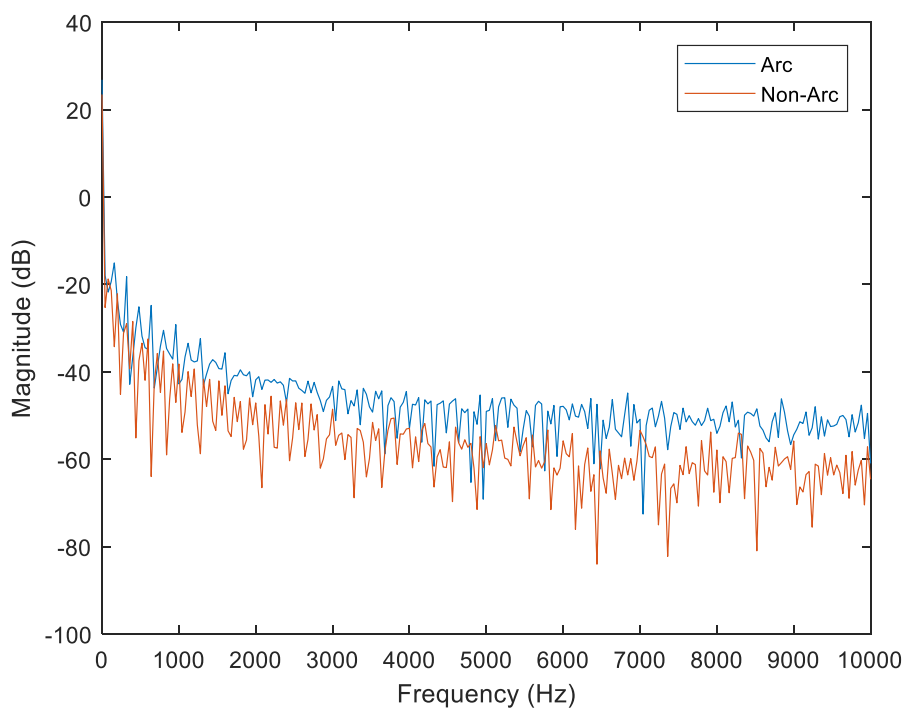

Figure 5: FFT spectrum (dB) of simulated arc current waveform 
simulation time, the circuit develops into a fully open-circuit condition.

An FFT of the simulated arc current waveform was performed to analyse changes in the frequency spectrum during both arcing and nonarcing conditions. A singular period of arcing/non-arcing behaviour was extracted from the time domain waveform, as indicated by the sample windows shown in Figure 3. The resultant single-sided amplitude spectrums are presented in Figure 4.

From the produced FFTs, it is clear that the majority of the signal energy is contained below the $3500 \mathrm{~Hz}$ range. The difference in magnitude is more pronounced when observed within in the logarithmic $(\mathrm{dB})$ scale in Figure 4. Both figures show clear distinctions between the arcing and non-arcing frequency spectrums, indicating that it may be exploited as an input parameter for arc fault detection algorithms.

\section{Series DC Arcing on Hardware Testbed}

A DC series Arc Fault Generation Unit (AFGU) was developed and utilised to create an intermittent arc fault within a laboratory environment to obtain experimental arc fault current data. Figure 6 shows the experimental hardware set-up including the linear actuator used to separate the copper electrodes, a DC stepper motor to precisely control the gap length and a Data Acquisitions system (DAQ) to collect the arc fault current data. The AFGU was controlled to provide a gap length of $0.1 \mathrm{~mm}$ at an intermittency of $5 \mathrm{~Hz}$ as observed in Figure 7. These variables were kept consistent during the simulation and hardware tests. Table 1 summarises the test set-up for both systems.

Table 1: Test Set-Up Summary

\begin{tabular}{|c|c|c|}
\hline Model & Simulation & Hardware \\
\hline Voltage (V) & $270 \mathrm{~V}$ & $28 \mathrm{~V}$ \\
\hline Resistance $(\Omega)$ & $18 \Omega$ & $1.65 \Omega$ \\
\hline Current (A) & $\sim 15 \mathrm{~A}$ & $\sim 17 \mathrm{~A}$ \\
\hline Gap Length & $0.1 \mathrm{~mm}$ & $0.1 \mathrm{~mm}$ \\
\hline $\begin{array}{l}\text { Intermittency } \\
\text { Frequency }\end{array}$ & $5 \mathrm{~Hz}$ & $5 \mathrm{~Hz}$ \\
\hline $\begin{array}{c}\text { Sampling Rate } \\
(\mathrm{kHz})\end{array}$ & $20 \mathrm{kHz}$ & $20 \mathrm{kHz}$ \\
\hline $\begin{array}{c}\text { Data Extraction } \\
\text { Window (ms) }\end{array}$ & $40 \mathrm{~ms}$ & $40 \mathrm{~ms}$ \\
\hline
\end{tabular}

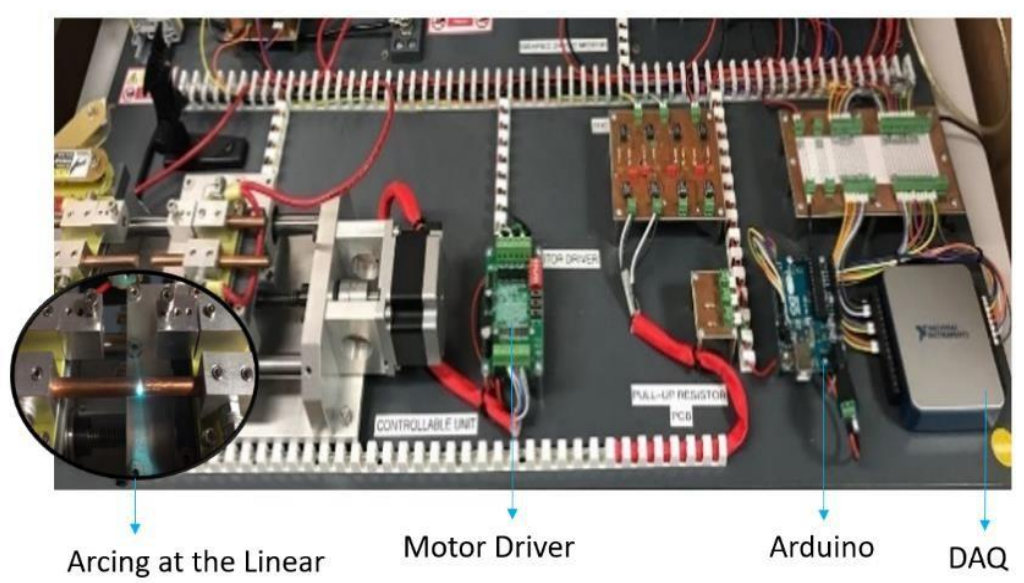

Actuator

Figure 6: The Arc Fault Generation Unit (AFGU)
As mentioned in the previous section, the simulation model was validated between $280 \mathrm{~V}$ to $800 \mathrm{~V}$, characterising arcing behaviour in a HVDC application. However, the hardware test rig was energised to $28 \mathrm{~V}$ with a resistive load rated to deliver similar current levels to provide a level of consistency between simulate on and hardware results. The sampling rate at which the test data was captured was also kept consistent at $20 \mathrm{kHz}$ with a data extraction window of $40 \mathrm{~ms}$.

Figure 7 shows the arc fault current data observed from the hardware test rig. Through a similar FFT analysis as conducted earlier in the section, Figures 8 and 9 show the changes in the frequency spectrum between arcing and non-arcing conditions.

Comparing the simulation and the hardware results similar trends were produced. The signal energy rise was observed below the $10 \mathrm{kHz}$ range, with significant activity witnessed under $\sim 3500 \mathrm{~Hz}$. This is in accordance with Parker et al. [11] who discussed frequency bands that contained essential information for discrimination of general arcing events and suggested that fractal sub-bands extending from 200 to $3500 \mathrm{~Hz}$ could be utilised for frequency based arc Fault Detection and Isolation (FDI). Also a study on DC series arc fault in photovoltaic systems for condition monitoring purposes [12]found that frequency bands below $50 \mathrm{kHz}$ exhibited a rise in arc floor noise and frequency contents below $5 \mathrm{kHz}$ are more sensitive towards change in air gap width.
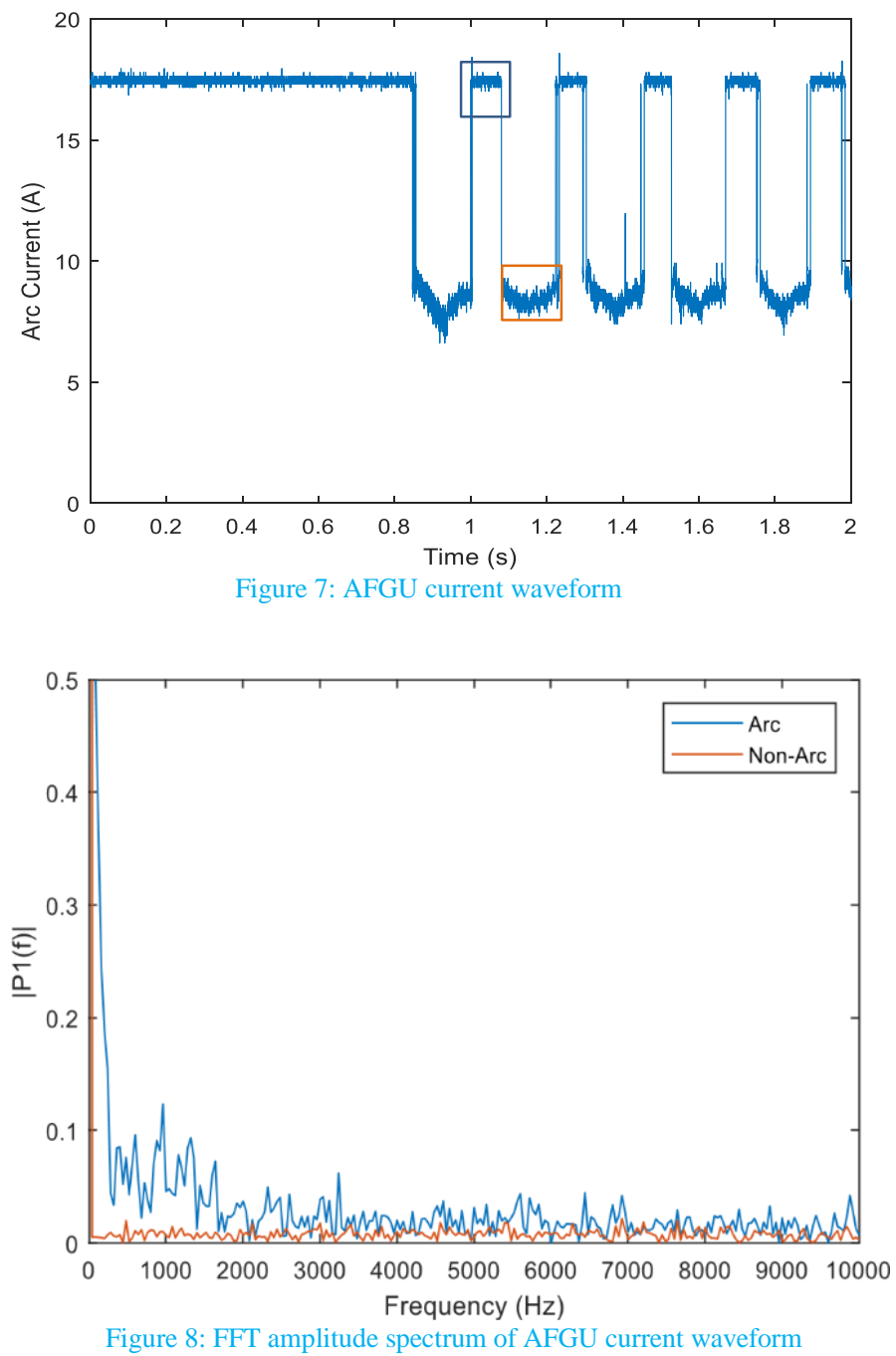

$10 / 19 / 2016$ 


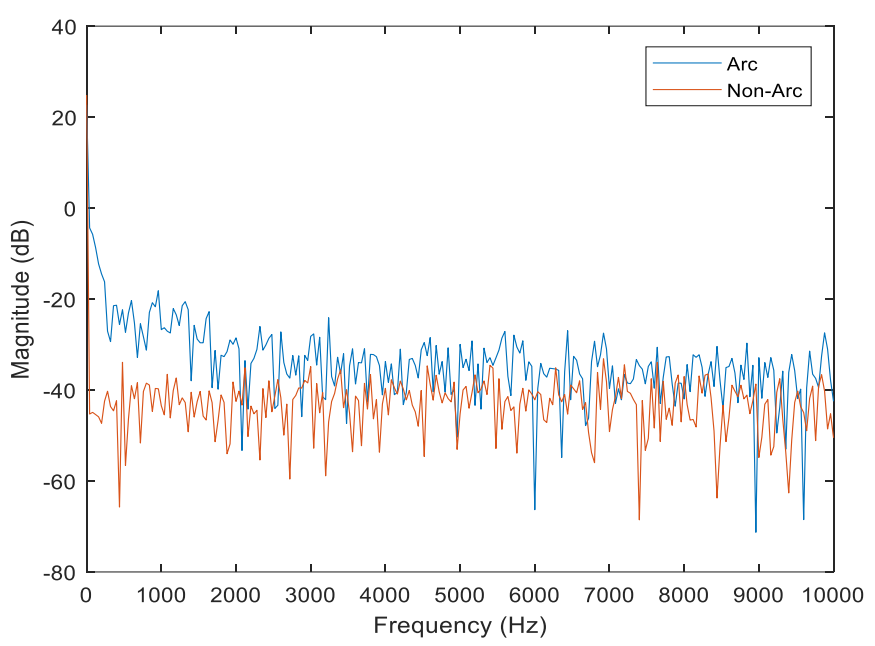

Figure 9: FFT spectrum (dB) of AFGU current waveform

Although, the exhibited distinct behaviour observed within the low frequency sub-bands can contribute to a basic threshold based detection method, the challenge lies in differentiating between a 'true arcing' condition and events that generate a similar profile such as load switching and inrush current. It is therefore common for detection methods to adapt to a domain that relies on frequency for feature extraction purposes while utilising larger data sets to observe for consistent trends. The following section highlights the detection methods that currently persist within the literature and the promising opportunities they present to detect a series DC arc fault.

\section{Review of Arc Fault Detection Methods}

The primary objective of an aircraft electrical protection system is to provide high levels of accuracy and sensitivity while maintaining reliability and minimising disruption to the critical loads. As the series arc fault condition proves to be highly intermittent in nature and reduces system current levels, it is necessary for a protection system that includes coverage of arc fault detection to discriminate between a true fault event and normal system transients. Deploying simplistic methods that assign constant threshold values for accurately detecting fault conditions and also account for transient behaviour is challenging. In general, aircraft protection systems should possess the following characteristics [9]:
- Dependable and Secure - accurately detect faults and minimise the probability of false alarms and non-detection.

- Real-time - detect faults online prior to the development of overcurrent faults.

- Non-intrusive - should not disrupt normal operation of the system.

- Low-cost - should be inexpensive while computational burden for data acquisition and signal processing should not be too heavy

This section describes numerous relevant general arc fault detection (AFD) methods that have been proposed in the literature. This review analyses the effectiveness of these methods in terms of meeting the characteristics required of an aircraft electrical protection system.

Figure 10 [13] summarises the classification of arc fault detection methods used for arc fault detection. For the purpose of low impedance arc fault detection (i.e. parallel arcing) conventional protection devices such as fuses, relays and CBs are utilised to provide sufficient protection coverage. High impedance, low fault current arc fault detection (AFD) methods are typically characterised as either nonelectrical or electrical. The following sections describe these methods in more detail.

\section{Non-Electrical AFD Methods}

Non-electrical AFD methods utilise special sensors which identify a measurement (pressure/thermal) [14] rise or a particular emission of light (infrared) [15] for detection. The acoustic signature [16] present in the arc, also makes a special case for detection in high powered arcs.

There are several limitations of mechanical detection methods, including the fact that the specialised sensors have high installation and maintenance costs. Also, non-electrical methods are not intended to provide complete system coverage since reliable detection requires the sensors being in close proximity to the arc [17]. The increased probability of false tripping raises another concern, as the sensors are highly susceptible to noise and have difficulty in discerning noise generated by other emitters on board an aircraft [13]. These limitations mean that non-electrical based AFD methods are unsuitable for series DC arc fault detection within aircraft.

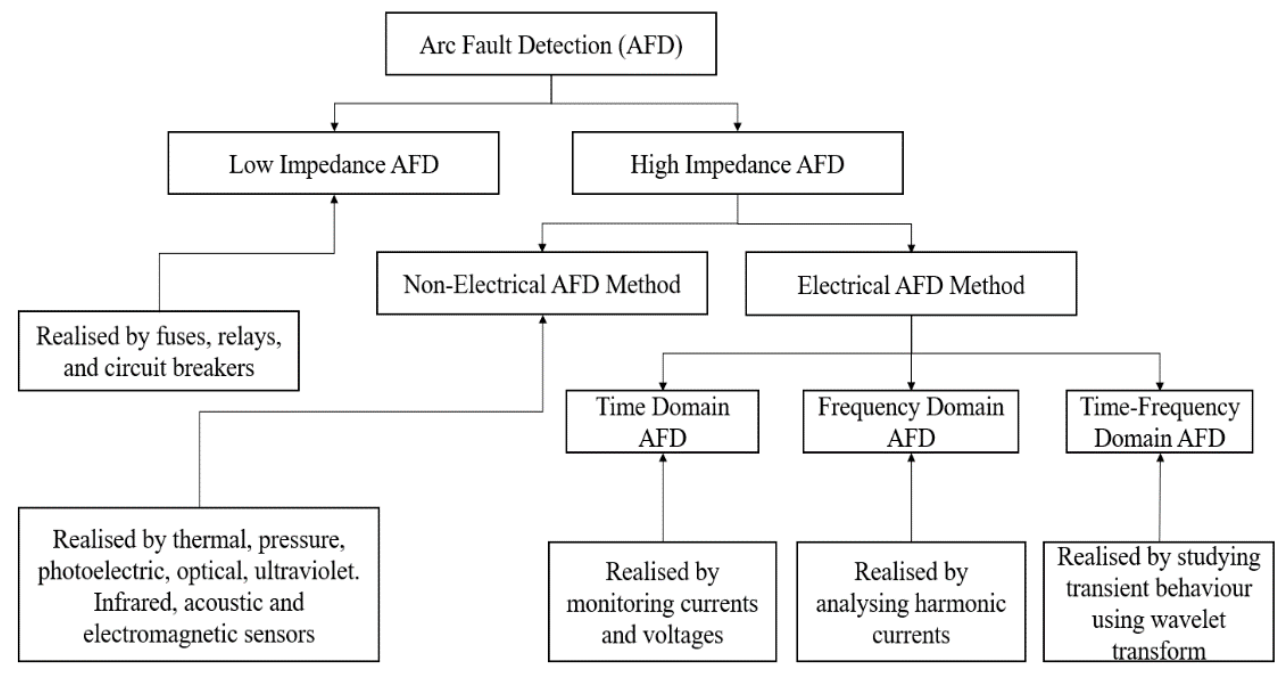

Figure 10: Classification of AFD Methods [13]

Page 5 of 11 


\section{Electrical AFD Methods}

Electrical AFD methods are defined as approaches based on algorithms that use feature extraction to analyse specific components to determine the presence of a fault. Observations made with variation in current, voltage and their electrical derivatives forms the basis for fault detection. Based on the domain of the feature extraction technique used, electrical FDI methods can be further categorised into the time domain, frequency domain and time-frequency domain [24].

\section{Time Domain}

Certain Time Domain AFD methods involves the analysis of differential current/voltage [18], imbalance of three phase current [19], voltage imbalance along a feeder [20] and arc fault energy [21]. Methods that employ voltage monitoring across a feeder for the purpose of detection and location of series faults, require voltage sensors installed across multiple measurement sites on a single conductor. The supplementary weight increase attests that these approaches not particularly suited for aircraft applications. However, the phase current imbalances and differential current characteristics detection methods are mainly only applicable to AC parallel arc faults. The lack of a ground plane, increases detection difficulty in aircraft EPS.

Blades [22] developed a device applied off-line to determine the AC voltage drop across a series of connections under load with no direct electrical contact to the conductors. Significant resistance observed at the series contacts was concluded to be symptomatic of a series arc fault. The device itself consists of a capacitive probe that clamps outside the insulation layer of the wire and a floating high-impedance meter at another node to measure a voltage difference. An increase in voltage past a pre-determined level would then further support the case for a series arc fault

Kilroy et al. [23] proposed a detection device and methodology that developed a signal resultant to the measurement of DC load current. A DC parallel arc event is identified by a software module when the difference between a maximum signal value and minimum signal value surpasses a certain threshold. The software module also averages signal values over multiple time periods and identifies a series DC arc in response to the average values surpassing a pre-determined threshold.

Andrea et al. [24] proposed a microprocessor based solid state power controller (SSPC) platform that detects arc faults. Each SSPC architecture protects its downstream load. The dynamic load requirements are pre-programmed and tripping occurs when the measured load current is outside a tolerable range. The multi-algorithm detection architecture developed, demonstrated the ability to detect approximately $75 \%$ of the series arc fault occurrences that were tested.

Guo et al. [30], develop a methodology that identifies the period of time between an immediate drop in the load current and arc ignition, defined as a precursor time. This enables the detection and isolation of potential DC series arcs before they actually occur. The equipment utilises a toroidal inductor that outputs a sharp increase in voltage, producing a fault alarm when there is an immediate current drop.

Dargatz et al. [31] designed a technique for photovoltaic (PV) systems that samples the DC current and voltage signature at the output terminals of a power converter which is then analysed for fault signatures. The difference is then averaged and values above a pre- determined threshold are suggestive of an arc fault event. A rapid rate of change (upwards of $0.1 \mathrm{~A}$ per microsecond) was determinant of a fault condition as typical transients exhibit a slower rate of change.

Another detection method for series arc faults was developed by Zuercher et al. [32] through the detection of DC load current or voltage undergoing a sudden drop. The load current is interrupted momentarily when a step decrease in current magnitude is detected. If the current does not return within a pre-determined margin to the decreased value prior to the interruption arcing is indicated. The current drifting upwards till the fault short circuits or a drifting downwards till it open circuits are other characteristics that's indicative of arc fault conditions. The power interruption to the critical loads or fault detection makes this method unsuitable for aircraft EPS application. A method by Sultana et al. [25] uses three overlapping running windows over the current waveform to detect DC series arcing conditions by utilising a simple change of mean scheme. The method was evaluated under loads varying from $400 \mathrm{~W}, 600 \mathrm{~W}$ and $800 \mathrm{~W}$. False detection is reduced by the methods capability of providing discrimination between load switching transients and a true arc fault. The authors also noted that the algorithm could be subject to further improvement by reducing the dependency on the current threshold values.

A detection technique for series DC arc faults using Rogowski coil [26] is proposed by Chen et al. The working band for this method is between $1 \mathrm{kHz}$ and $10 \mathrm{MHz}$ with a sensitivity of $0.5 \mathrm{~V} / \mathrm{A}$. An initial spike observed in the waveform measured by the device is then utilised to set a time domain threshold to determine a fault. The technique promises a high sampling and processing accuracy through a reduction in the quantisation error.

Time domain arc FDI limitations mainly arise from the process of determining thresholds and defining features that actively distinguish between true arcing and events that produce similar load characteristic profiles such as load inrush and switching transients.

\section{Frequency Domain}

Numerous AFD methods have been proposed in the literature based on analysis of voltage and current frequency spectra. The Fast Fourier Transform (FFT) efficiently translates the time domain signals to the frequency domain. The Discrete Fourier Transform (DFT) possess the ability to decompose signals into spectral components. The harmonic voltage and or current data can be analysed to provide discrimination between normal transient and fault conditions for the purpose of arc fault detection.

Scott et al. [27] propose a system that determines the presence of broadband noise signals in load current through the monitoring of one or more conductors and develops an input signal that is representative of electrical signal condition for application within aircraft EPS. Evidence of a broadband spectrum being present with the energy levels of harmonics for pre-set range of frequencies is then indicative of a fault. The indication of an arc is then proceeded by an output trip signal that could directly or indirectly trip a circuit interruption device.

Kwon et al. [28] propose a similar method for high impedance fault detection that relies on the incremental variance of normalised even order harmonic ratios. The criterion was developed in accordance to the unsymmetrical behaviour observed in the fault current waveform.

Xiaochen et al. [29] utilise a method that detects AC series arc faults based on the Mahalanobis distance; the distance between two points in 
multivariate space. Characteristics of both normal and arc current are analysed to obtain the Mahalanobis distance relative to the supply frequency. The feasibility of the method was verified on a $115 \mathrm{~V}$ system with a purely resistive load and exhibited no false trips under loading and de-loading conditions.

Kojori et al. [33] propose a real-time analysis method of load current through the implementation of a moving/sliding DFT window that performs steady state component monitoring. An amplitude variation profile in the DC component is deemed to be conclusive of an arc fault being present. The variation within the data is extracted by counting the number of maxima over a period in comparison with a set threshold. The method combined with the measurement variations observed on load current distortion was found to increase the accuracy of the fault detection.

Ohta and Isoda [34] designed a device on similar principles that acquires voltage or current time series data and implements frequency analysis to generate data based on the multitude of frequency components to evaluate the manifestation of an arc. The observation of the evaluation value exceeding a pre-determined arc judgement threshold value was also deemed to support the identification of the symptoms of an arc being present in the system.

Momoh and Button [35] introduce an AFD method which utilises FFT for the decomposition of the monitored signals (voltage and current) in conjunction with a back propagation algorithm of artificial neural networks (ANN) to detect arcing faults. The system was shown to be able to perform detection over several system conditions and proved to be fast and accurate, making it ideal for real-time applications within aerospace applications. However, challenges remain surrounding the definition of ANN model structure.

Gao et al. [36] samples current signal data during a DC series arc through field experiments on a PV power station and utilises feature extraction both in time and frequency domains to classify arcing and non-arcing. This data was then used to train Bayesian support vector machines (BSVM) with two selected features and achieved the classification of arcing and non-arcing by a separating line in the feature space. The performance of classification was evaluated by defining and calculating the rate of correct classification, false alarm and missed detection. The results produced promise high accuracy.

Chen et al. [37] propose a pattern matching algorithm to detect low voltage series DC arcs. An FFT is implemented for the decomposition of the monitored current signal. Dynamic time warping (DTW) measures the input and arc model. The method proves to be effective at detecting and classifying the arc fault.

A related study on DC series arc faults in PV systems [12] by Lu et al. for condition monitoring purposes, examined several arc models through a heuristic approach. The investigation of arc noise features under the electrode separation region found an increase in the arc noise floor following an arc fault occurrence, specifically for frequency bands below $50 \mathrm{kHz}$. The study also found that frequency components below $5 \mathrm{kHz}$ are highly sensitive to the air gap width. These characteristics can be utilised for detection purposes.

Although many frequency domain based AFD methods have been proposed in the literature, their main disadvantages lie in the fact that the FFT does not have the capability to associate the time at which the harmonic frequencies are present. FFT proves to be an ideal platform for stationary and continuous fault conditions. However, for highly transient signals [41] such as series DC arc faults the absence of time Page 7 of 11 information may limit the accuracy of the purely frequency domain based methods.

\section{Time-Frequency Domain}

Time-Frequency Domain arc FDI methods determine how the frequency behaviour changes with time. These often employ the Short Time Fourier Transform (STFT), through the utilisation of a sliding window that provides the determination of the sinusoidal frequency and the phase contents of a local section of a signal, with variation in time.

Chen and Xiong [38], utilise STFT to detect the varying characteristic frequency bands produced by arc noises. The disadvantage associated with STFT is the compromise that has to be undergone when the prioritisation of time resolution vs. frequency resolution is a factor. A shorter window length lends itself to fast transient signals, providing a higher resolution in locating time domain behaviour whereas a longer window length provides a higher frequency resolution but the information is averaged across a longer time frame and potentially smearing out non-stationary behaviour.

The Wavelet Transform (WT) can provide the frequency of a signal as well as the time associated with each frequency. For cases associated with highly transient and non-stationary signals, WT proves to be ideal. The WT has a leverage over the STFT as the analysing 'window' is variable in size. The general challenge associated within WT based detection methods is the evaluation of generated wavelet coefficients for the purpose of determining the presence of a fault. Advanced detection systems utilise intelligent techniques applied with WT features to determine the probability of fault presence.

Yunmei et al. [39] use the evaluation of time-frequency signatures of series arc fault current, realised through WT to detect DC arc faults in low voltage $28 \mathrm{VDC}$ network. The authors of the research concluded that calculation of five consecutive wavelet coefficients produced discrimination between fault condition and load transients under normal conditions.

A method based on both time and time-frequency domain features for the application in DC microgrid networks is presented by Yao et al. in [40]. Implemented through a DSP board, the detection algorithm calculates the maximum and minimum values and the corresponding difference, from the time domain arc current data through a specified $25 \mathrm{~ms}$ window. In combination with the RMS values of wavelet coefficients within the $25-50 \mathrm{kHz}$ fractal sub-band, flagging and indication of the presence of a series arc fault is then achieved. Although the authors decided on four consecutive flags to be accurately indicative of faults while avoiding nuisance trips, they do not determine whether this number is optimal across systems with varying system configurations and transients.

Telford et al. [41] propose a machine learning technique that utilises feature extraction from the time and time-frequency domain along with Hidden Makarov Models (HMM) to discriminate between nominal and arc fault behaviour. The implementation of the DWT coefficients within the HMM system result in high diagnostic accuracy while accommodating a variety of system operating conditions.

Table 2 summarises the detection methodologies that have been discussed in the paper in terms of particular area of application, fault category and the power system type. 


\begin{tabular}{|c|c|c|c|c|c|}
\hline AFD Method & Domain & Techniques/Study & Application & Series/Parallel & AC/DC \\
\hline \multirow{4}{*}{ Non-Electrical } & \multirow{4}{*}{-} & Thermal [14] & Residential & Series/Parallel & $\mathrm{AC}$ \\
\hline & & Pressure [14] & Submarines & Series/Parallel & DC \\
\hline & & Infrared [15] & Switchgear/Power Systems & Series/Parallel & $\mathrm{AC} / \mathrm{DC}$ \\
\hline & & EM Sensors [17] & LV Systems & Series/Parallel & $\mathrm{AC}$ \\
\hline \multirow{21}{*}{ Electrical } & \multirow{8}{*}{ Time } & Voltage drop [22] & Aircraft EPS & Series & $\mathrm{AC}$ \\
\hline & & Statistics [23] & Software & Series/Parallel & DC \\
\hline & & SSPC [24] & Circuit Interrupters & Series/Parallel & $\mathrm{AC} / \mathrm{DC}$ \\
\hline & & Overlapping window[25] & DC Grids & Series & DC \\
\hline & & Rogowski coil [26] & Circuit Interrupters & Series & DC \\
\hline & & Current drift [32] & Circuit Interrupters & Series/Parallel & $\mathrm{DC}$ \\
\hline & & Rate of current change [31] & PV & Series/Parallel & $\mathrm{DC}$ \\
\hline & & Current drop [30] & Circuit Interrupters & Series & $\mathrm{DC}$ \\
\hline & \multirow{9}{*}{ Frequency } & Broadband noise [27] & Aircraft EPS & Series/Parallel & $\mathrm{AC}$ \\
\hline & & Harmonic ratios [28] & Power Substation & Parallel & $\mathrm{AC}$ \\
\hline & & Mahalanobis [29] & Circuit Interrupters & Series & $\mathrm{AC}$ \\
\hline & & General arc FDI [11] & Aircraft EPS & Series/Parallel & $\mathrm{AC} / \mathrm{DC}$ \\
\hline & & Sliding DFT window [33] & Spacecraft EPS & Series/Parallel & $\mathrm{AC} / \mathrm{DC}$ \\
\hline & & Frequency component [34] & Aircraft EPS & Series & $\mathrm{DC}$ \\
\hline & & ANN [35] & Spacecraft EPS & Series & $\mathrm{DC}$ \\
\hline & & BSVM [36] & PV & Series & $\mathrm{DC}$ \\
\hline & & DTW [37] & AC Distribution & Series & DC \\
\hline & \multirow{4}{*}{ Time-Frequency } & STFT [38] & PV & Series & DC \\
\hline & & WT [39] & Circuit Interrupters & Series/Parallel & DC \\
\hline & & DSP [40] & PV & Series & DC \\
\hline & & HMM [41] & MEA EPS & Series & DC \\
\hline
\end{tabular}

\section{Application of AFD Methods in MEA}

It has been shown that AFD methods utilise either a deterministic or a probabilistic approach, or in certain cases a combination of the two. By definition, a deterministic solution is designed with the intention of arriving at a singular solution/outcome whereas a probabilistic model provides a distribution of the possible outcomes with a certain measure of likelihood. Within the context of MEA EPS, the detection methodology utilized would need to meet predetermined standards that promote safety and reliability while optimising the balance between sensitivity and security. Although it could be argued that no single system is $100 \%$ deterministic, in a scenario where safety is paramount, a deterministic method might hold the preference for detection purposes by promoting a higher degree of certainty. The shortcoming, is the resultant slower detection times as a result of it being primarily time-domain dominant. On the contrary, a probabilistic approach to detection does have its place as it has the potential to detect an arc

before it occurs. Additionally, unlike deterministic methods, there is less dependency on pre-existing knowledge of the system and its conditions for probabilistic methods to perform to their full potential.
There is an opportunity to further explore the roles of both deterministic and probabilistic methods for MEA applications. For example, whilst most deterministic AFD methods explored in the literature utilise single point measurements, the compact physical nature of an MEA power system and pre-existing sensor, communications and system control infrastructure encourages the exploration of multi-device and multi-method deterministic AFD approaches. These may achieve higher levels of performance for particular MEA applications than the methods presented in the literature.

Additionally, whilst the challenge of achieving flight certification has heavily restricted the use of probabilistic methods in existing aircraft to date, there may be the opportunity to employ these to protect non flight-critical electrical loads or network sections, or to combine these with deterministic methods so that a suitably high degree of operational certainty can still be achieved. In this manner, the authors believe that an MEA-specific focus to AFD research could yield significant benefits. 


\section{Summary/Further Work}

The paper has described series DC arc fault features by examining frequency spectrum changes implemented through FFT analysis. Series DC arc faults were iterated both through a simulation model and by utilizing an Arc Fault Generation Unit. Arcing and non-arcing characteristics were distinguishable on the power magnitude spectrum. Additionally, frequencies under $3500 \mathrm{~Hz}$ exhibited substantial changes which could be extracted for detection purposes, as supported by existing literature. Arc fault detection methods with relevance to series or parallel DC arcing were also reviewed, considering in particular the application of unique constraints that application to an MEA EPS would bring.

The authors intend to develop the work presented further, by undertaking additional behavioural analysis on a time-frequency domain utilizing STFT (Short Time Frequency Transform) or DWT (Discrete Wavelet Transform) approach in order to identify specific frequency bands that could aid in fault detection. The authors also intend to explore the use of purely deterministic and coupled probabilistic-deterministic methods to address the unique challenges of method application to MEA platforms.

\section{Contact Information}

The authors are with the Institute for Energy and Environment, Department of Electronics and Electrical Engineering, University of Strathclyde, Technology and Innovation Centre, 99 George Street, Glasgow, UK, G1 1RD. E-mail: jeffy.johny@strath.ac.uk

\section{Acknowledgements}

This work was carried out as part of the Rolls-Royce University Technology Centre programme.

\section{References}

[1] M. E. Baran and N. R. Mahajan, "DC distribution for industrial systems: opportunities and challenges," IEEE Trans. Ind. Appl., vol. 39, no. 6, pp. 1596-1601, 2003.

[2] D. J. Hammerstrom, "AC versus DC distribution systemsdid we get it right?," 2007 IEEE Power Eng. Soc. Gen. Meet. PES, pp. 1-5, 2007.

[3] A. Yaramasu, Y. Cao, G. Liu, and B. Wu, "Aircraft electric system intermittent arc fault detection and location," IEEE Trans. Aerosp. Electron. Syst., vol. 51, no. 1, pp. 40-51, 2015.

[4] C. E. Restrepo, "Arc Fault Detection and Discimination Methods," Electr. contacts - 2007, 53rd ieee holm Conf., 2007.

[5] A. D. Stokes and W. T. Oppenlander, "Electric arcs in open air," J. Phys. D. Appl. Phys., vol. 24, no. 1, pp. 26-35, 1991.

[6] P. K. Kuhn, C. Furse, and P. Smith, "Locating Hidden Hazards in Electrical Wiring," Aged Electr. Syst. Res. Appl. Symp., pp. 1-8, 2006.

[7] Brusso, Barry C. "History of Aircraft Wiring Arc-Fault Protection [History]." IEEE Industry Applications Magazine 23, no. 3 pp.6-11 2017.
J. Li, D. W. P. Thomas, M. Sumner, E. Christopher, and Y. Cao, "Series Arc fault studies and modeling for a DC distribution system," Asia-Pacific Power Energy Eng. Conf. APPEEC, pp. 1-6, 2013.

[9] R. Telford, "Novel Methods for Improving Fault Protection $\&$ Health Management within Advanced Aircraft Electrical Power Systems," no. April, 2017.

[10] Uriarte, Fabian M., Angelo L. Gattozzi, John D. Herbst, Hunter B. Estes, Thomas J. Hotz, Alexis Kwasinski, and Robert E. Hebner. "A DC arc model for series faults in low voltage microgrids." IEEE Transactions on smart grid 3, no. 4, 2012.

[11] M. T. Parker, H. M. H. Jr, J. J. Keenan, and L. P. Benoit, "Electric arc monitoring systems," no. US6772077 B1. Aug2004.

[12] Lu, Shibo, B. T. Phung, and Daming Zhang. "Study on DC series arc fault in photovoltaic systems for condition monitoring purpose." In Universities Power Engineering Conference (AUPEC), 2017 Australasian, pp. 1-6. IEEE, 2017.

[13] G. Liu, Y. N. Cao, Y. Liu, and Z. Liu, "A Survey on Arc Fault Detection and Wire Fault Location for Aircraft Wiring Systems," SAE Int. J. Aerosp., vol. 5, no. 2, pp. 579-589, 2012.

[14] H. B. Land, "Sensing Switchboard Arc Faults," IEEE Power Eng. Rev., vol. 22, no. 4, pp. 18-27, 2002.

[15] L. Kumpulainen, G. A. Hussain, M. Lehtonen, and J. A. Kay, "Preemptive arc fault detection techniques in switchgear and controlgear," IEEE Trans. Ind. Appl., vol. 49, no. 4, pp. 1911-1919, 2013.

[16] K. Yang, R. Zhang, J. Yang, C. Liu, S. Chen, and F. Zhang, "A novel arc fault detector for early detection of electrical fires," Sensors (Switzerland), vol. 16, no. 4, pp. 1-24, 2016.

[17] C. Vasile and C. Ioana, "Arc Fault Detection \& Localization by Electromagnetic-Acoustic Remote Sensing," IOP Conf. Ser. Mater. Sci. Eng., vol. 198, no. 1, pp. 0-1, 2017.

[18] Pellon, Christian V., Christopher A. Nicolls, and Michael T. Parker. "Low cost arc fault detection technique." U.S. Patent 7,400,481, issued July 15, 2008.

[19] C. W. Hull, "Apparatus for production of three-dimensional objects by stereolithography," US Pat. 4,575,330, vol. 1, no. 12, pp. 1-16, 1986.

[20] E. C. Senger, W. Kaiser, J. C. Santos, and P. M. S. Burt, "Communication," Power, vol. 15, no. 2, pp. 525-530, 2000 .

[21] Zuercher, Joseph C., and David L. McClanahan. "Apparatus and method for real time determination of arc fault energy, location and type." U.S. Patent 7,068,045, issued June 27, 2006.

[22] Blades, Frederick K. "Series arc fault diagnostic for aircraft wiring." U.S. Patent 6,882,158, issued April 19, 2005.

[23] D. G. Kilroy and W. H. Oldenburg, "DC arc fault detection and protection," no. EP1796238 B1. Jan-2013.

[24] J. Andrea, O. Zirn, and M. Bournat, "Principle of Arc Fault Detection for Solid State Power Controller," 2012 IEEE 
58th Holm Conf. Electr. Contacts, pp. 1-6, 2012.

[25] Q. Sultana, P. Mishra, and S. Chary, "Novel control methodology for detecting series arc in DC circuits," 2017 IEEE 2nd Int. Conf. Direct Curr. Microgrids, ICDCM 2017, pp. 12-17, 2017.

[26] Shiying Chen ; Lingyu Zhu; Shengchang Ji ; Xiaojun Liu, "Detection of series DC arc fault using rogowski coil," in 2017 IEEE Conference on Electrical Insulation and Dielectric Phenomenon (CEIDP).

[27] G. W. Scott and K. B. Wong, "Arc fault detection for aircraft," no. US6625550 B1. Sep-2003.

[28] W. H. Kwon and G. W. Lee, "Power System Relaying High Impedance Fault Detection Utilizing Incremental Variance Of Normalized Even Order Harmonic Power," IEEE Power Eng. Rev., vol. 11, no. 4, pp. 58-59, 1991.

[29] X. Cai, W. Li, Q. Sun, and M. Zhen, "AC arc fault detection based on mahalanobis distance," 15th Int. Power Electron. Motion Control Conf. Expo. EPE-PEMC 2012 ECCE Eur., pp. 1-6, 2012.

[30] S. Y. Guo, J. L. J. III, and A. S. Dooley, "DC arc detection and prevention circuit and method," no. US6683766 B1. Jan-2004.

[31] M. Dargatz and M. Fornage, "Method and apparatus for detection and control of dc arc faults," no. US8179147 B2. May-2012.

[32] J. Zuercher, J. Hastings, E. Hetzmannseder, J. Pardee, and C. Tennies, "Detection of arcing in dc electrical systems," no. US20040027749 A1. Feb-2004.

[33] H. Kojori, C. Li, and F. Dawson, "Method and apparatus for generalized arc fault detection," no. US20060203401 A1. Sep-2006.

[34] Y. Ohta and H. Isoda, "ARC Detecting device and aircraft equipped therewith,” no. EP2120306 A2. Nov-2009.

[35] Momoh, James A., and Robbert Button. "Design and analysis of aerospace DC arcing faults using fast fourier transformation and artificial neural network." In Power Engineering Society General Meeting, 2003, IEEE, vol. 2, pp. 788-793. IEEE, 2003.

[36] Y. Gao, J. Dong, Y. Sun, Y. Lin, and R. Zhang, "PV arcfault feature extraction and detection based on bayesian support vector machines," Int. J. Smart Grid Clean Energy, pp. 283-290, 2015.

[37] M. Chen et al., "Detection method of low voltage series DC arc based on the pattern matching algorithm," 2017 20th Int. Conf. Electr. Mach. Syst. ICEMS 2017, 2017.

[38] S. Chen, X. Li, and J. Xiong, "Series Arc Fault Identification for Photovoltaic System Based on Time-Domain and TimeFrequency-Domain Analysis," IEEE J. Photovoltaics, vol. 7, no. 4, pp. 1105-1114, 2017.

[39] G. Yunmei, W. Li, W. Zhuoqi, and J. Binfeng, "Wavelet packet analysis applied in detection of low-voltage DC arc fault," 2009 4th IEEE Conf. Ind. Electron. Appl. ICIEA 2009, vol. 2, no. 1, pp. 4013-4016, 2009.

[40] X. Yao, L. Herrera, and J. Wang, "A series DC arc fault detection method and hardware implementation," in 2013
Twenty-Eighth Annual IEEE Applied Power Electronics Conference and Exposition (APEC), 2013, pp. 2444-2449.

[41] R. D. Telford, S. Galloway, B. Stephen, and I. Elders, "Diagnosis of Series DC Arc Faults-A Machine Learning Approach," IEEE Trans. Ind. Informatics, vol. 13, no. 4, pp. 1598-1609, 2017.

\section{Definitions/Abbreviations}

\begin{tabular}{|c|c|}
\hline MEA & More Electric Aircraft \\
\hline DC & Direct Current \\
\hline $\mathbf{A C}$ & Alternating Current \\
\hline CB & Circuit Breaker \\
\hline AFCB & Arc Fault Circuit Breaker \\
\hline dB & Decibel \\
\hline FGU & Fault Generation Unit \\
\hline HVDC & $\begin{array}{l}\text { High Voltage Direct } \\
\text { Current }\end{array}$ \\
\hline LVDC & $\begin{array}{l}\text { Low Voltage Direct } \\
\text { Current }\end{array}$ \\
\hline FDI & $\begin{array}{l}\text { Fault Detection \& } \\
\text { Isolation }\end{array}$ \\
\hline AFD & Arc Fault Detection \\
\hline SSPC & $\begin{array}{l}\text { Solid State Power } \\
\text { Controller }\end{array}$ \\
\hline FFT & Fast Fourier Transform \\
\hline DFT & $\begin{array}{l}\text { Discrete Fourier } \\
\text { Transform }\end{array}$ \\
\hline DWT & $\begin{array}{l}\text { Discrete Wavelet } \\
\text { Transform }\end{array}$ \\
\hline WT & Wavelet Transform \\
\hline STFT & $\begin{array}{l}\text { Short Time Fourier } \\
\text { Transform }\end{array}$ \\
\hline DSP & Digital Signal Processor \\
\hline $\mathbf{A N N}$ & $\begin{array}{l}\text { Artificial Neural } \\
\text { Network }\end{array}$ \\
\hline BSVM & $\begin{array}{l}\text { Bayesian Support } \\
\text { Vector Machine }\end{array}$ \\
\hline
\end{tabular}

Page 10 of 11

$10 / 19 / 2016$ 
HMM

RMS

Page 11 of 11

Hidden Markov Models

Root Mean Square

$10 / 19 / 2016$ 